\title{
Left Internal Mammary-to-Left Circumflex Coronary Artery Collateral Pathway in a Patient With Occluded Left Circumflex Artery
}

\author{
Takeshi Yamamoto, MD; Yasuhira Kasagami, MD; Takayoshi Ohba, MD; \\ Kazunori Saeki, MD; Masafumi Kanemura, MD; \\ Kazuo Munakata, MD*; Teruo Takano, MD*
}

\begin{abstract}
A unique case of left internal mammary artery-to-circumflex coronary artery collateral pathway, which was conceivably encouraged to develop by coronary occlusion. The pathway provided sufficient collateral perfusion to preserve the local myocardial performance. (Jpn Circ J 2001; 65: 753-754)
\end{abstract}

Key Words: Coronary artery disease; Internal mammary-coronary artery anastomosis

$\mathbf{T}$ he occurrence of intercoronary collaterals is well known, and collateral pathways between the coronary artery and extracardiac systemic arteries have also been demonstrated by postmortem angiographic studies, 1,2 but are rarely detected in living subjects?, ${ }^{4} \mathrm{We}$ describe a unique case of a spontaneously developed collateral pathway from the left internal mammary artery (IMA) to the left circumflex artery (LCX) that provided sufficient myocardial perfusion to preserve the local myocardial function.

\section{Case Report}

A 75-year-old Japanese woman was admitted to Kitamurayama General Hospital because of severe dyspnea. An electrocardiogram revealed first-degree atrioventricular block, elevation of the ST segment in leads II, III and aVF, and depression of the ST segment in leads I, $\mathrm{VV}_{\mathrm{L}}, \mathrm{V}_{5}$ and V6. The white blood cell count was 9,800 $\mu 1$, creatine kinase (CK) 600 IU/L, CK-MB 77 IU/L. Thus she was diagnosed as acute inferior myocardial infarction. Twenty-one days later, cardiac catheterization was performed and the left ventriculogram showed mild hypokinesis of the inferior segment (Fig 1). A coronary angiogram revealed the total occlusion of the proximal segment of the right coronary artery (RCA) that was responsible for the infarction (Fig 2). The proximal segment of the left anterior descending artery (LAD) had 99\% stenosis, and the middle segment of the LCX was also totally occluded (Fig 3). During arteriography of the left IMA for potential surgical treatment, an enlarged pericardial branch was demonstrated, arising from the upper portion of the left IMA and communicating with the LCX through retrograde filling of the left atrial branch (Fig 4). Because of the difficulty in holding the catheter at

(Received December 4, 2000; revised manuscript received February 13, 2001; accepted February 22, 2001)

Department of Internal Medicine, Kitamurayama General Hospital, Yamagata and *First Department of Internal Medicine, Nippon Medical School, Tokyo, Japan

Mailing address: Takeshi Yamamoto, MD, First Department of Internal Medicine, Nippon Medical School, 1-1-5 Sendagi, Bunkyoku, Tokyo 113-8603, Japan the ostium of the left IMA, we could not obtain an arteriogram in the orthogonal view, which we supposed would confirm the existence of a communication between the left

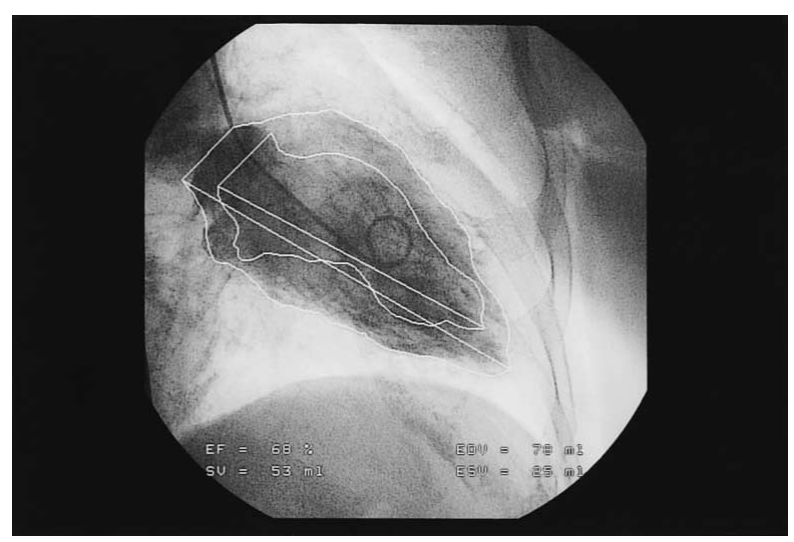

Fig 1. Left ventriculogram in the right anterior oblique view showing mild hypokinesis of the inferior segment.

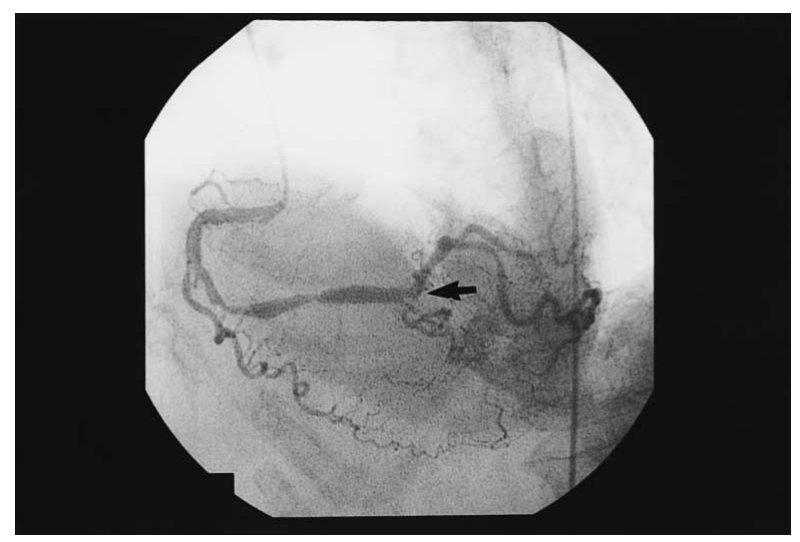

Fig 2. Right coronary arteriogram in the left anterior oblique view showing total occlusion in the proximal segment of the posterior descending artery (arrow) with collateral from the right ventricular branch. 


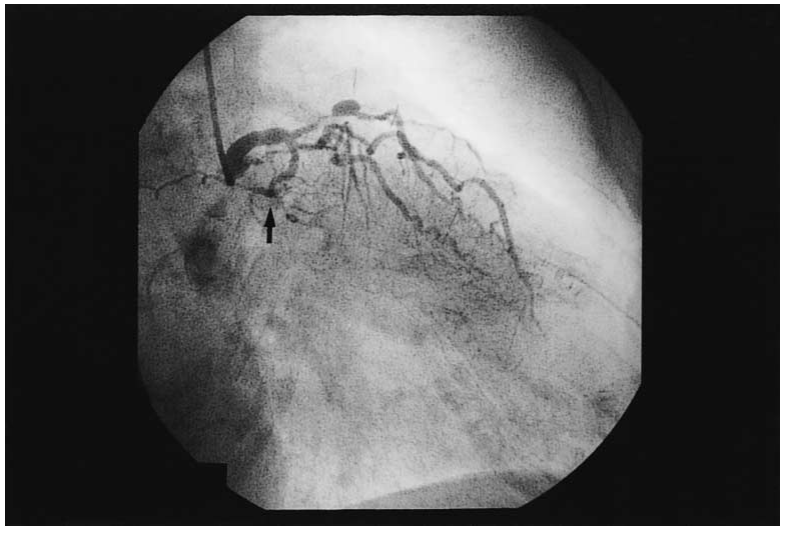

Fig 3. Left coronary arteriogram in the right anterior oblique view showing total occlusion in the middle segment of the left circumflex artery (arrow)

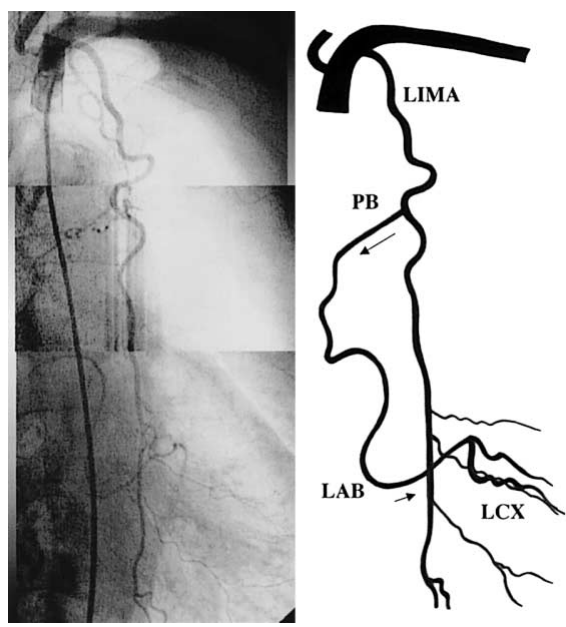

Fig 4. Left internal mammary arteriogram and diagram outlining the left internal mammary-to-left circumflex coronary artery communication. The slightly enlarged pericardial branch (PB) of the left internal mammary artery (LIMA) communicated with the left circumflex artery (LCX) through retrograde filling of the left atrial branch (LAB).
Vertical long-axis
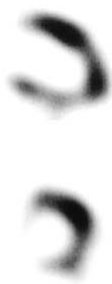

Vertical short-axis

Horizontal long-axis

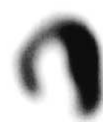

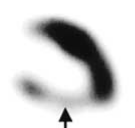

4
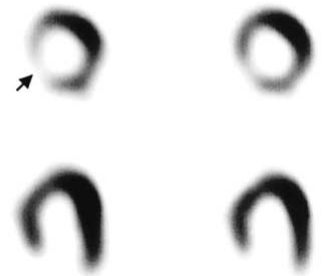

Fig 5. Myocardial perfusion imaging with thallium-201 singlephoton emission computed tomography shows that the inferior wall was mildly hypoperfused (arrows), while the posterolateral wall was normally perfused.
IMA and the LCX. Myocardial perfusion imaging with thallium-201 single-photon emission computed tomography demonstrated that the inferior wall was hypoperfused, whereas the posterolateral wall was well perfused (Fig 5). This observation suggested that the collateral flow from the left IMA was sufficient for preserving the left ventricular performance in spite of the occluded LCX.

\section{Discussion}

Coronary collateral pathways develop as a sequel to concomitant occlusive coronary artery disease. Collaterals probably develop from extracardiac vessels to the coronary arteries $^{1-4}$ and the potential for development of coronary collaterals from the IMA has been recognized for some time, and formed the basis for attempted myocardial revascularization by IMA ligation5,6 In the study by Moberg on extracardiac-coronary anastomoses using postmortem angiography of 49 cadavers, contrast media reached the atrial branches of the coronary arteries on IMA injection in 15 cases, and the ventricular branches in 4 cases that all had coronary artery disease? It is conceivable that myocardial ischemia was the main stimulus for the development of those collaterals to the ventricle, but it is important to note in the present case that myocardium that was supposed to be perfused by the LAD or LCX was salvaged in spite of severe stenosis in these coronary arteries, while myocardium perfused by the RCA without collateral perfusion resulted in infarct.

The extracardiac vessels can communicate with the coronary arteries when they reach the site of pericardial reflection and then communicate either via the aortic vasa vasorum or through the atrial branches. Therefore, the development of the collaterals in the present case may have resulted from the distribution of the IMA at birth. Only 2 living cases with this unique communication have been reported $^{3,4}$ and in those patients, the collateral pathway was from the pericardial branch of the right IMA via the sinus node branch to the RCA, which was totally occluded. The present patient is the first live case with a collateral pathway from the left IMA to the LCX.

The possibility of these coronary collateral pathways from the IMA should be kept in mind during coronary angiography if the absence of a major coronary artery cannot be otherwise explained.

\section{References}

1. Moberg A: Anastomoses between extracardiac vessels and coronary arteries. I. Via bronchial arteries. Acta Radiol Diagn 1967; 6: $177-$ 192

2. Moberg A: Anastomoses between extracardiac vessels and coronary arteries. II. Via internal mammary arteries. Acta Radiol Diagn 1967; 6: $263-272$

3. Singh RN, Varat MA: Acquired internal mammary-to-coronary artery communication. Cathet Cardiovasc Diagn 1982; 8: 281-285

4. Kajinami K, Takekoshi N, Yoshio H: Internal mammary-to-coronary artery communication in a patient with occluded right coronary artery. Am Heart J 1993; 125: 1428-1430

5. Blair CR, Roth RF, Zintel HA: Evaluation of internal mammary artery ligation for relief of angina pectoris. Surg Forum 1957; 8: 345-348

6. Glover RP, Devila JC, Kyle RH, Brard JC Jr, Trout RG, Kitchell JR: Ligation of the internal mammary arteries as a means of increasing blood supply to the myocardium. J Thorac Surg 1957; 34: 661-678 